\title{
STARLIKE FUNCTIONS
}

\author{
CARL P. MCCARTY
}

\begin{abstract}
Let $\mathscr{S}^{*}[\alpha]$ denote the class of functions $f(z)=z+$ $\sum_{n=2}^{\infty} a_{n} z^{n}$ analytic in $|z|<1$ and for which $\left|z f^{\prime}(z)\right| f(z)-1 \mid<1-\alpha$ for $|z|<1$ and $\alpha \in[0,1)$. Sharp results concerning coefficients, distortion, and the radius of convexity are obtained. Furthermore, it is shown that $\sum_{n=2}^{\infty}[(n-\alpha) /(1-\alpha)]\left|a_{n}\right|<1$ is a sufficient condition for $f(z) \in \mathscr{S}^{*}[\alpha]$.
\end{abstract}

1. Introduction. Suppose that $f(z)=z+\sum_{n=2}^{\infty} a_{n} z^{n}$ is analytic and $\operatorname{Re}\left\{z f^{\prime}(z) \mid f(z)\right\}>\alpha$ for $|z|<1$ and $\alpha \in[0,1)$, then $f(z)$ is called starlike of order $\alpha$, denoted by $f(z) \in \mathscr{S}_{\alpha}^{*}$. It was shown by Schild [7] that for $f(z) \in$ $\mathscr{S}_{\alpha}^{*}$ the domain of values of $\left\{z f^{\prime}(z) \mid f(z)\right\}$ is the circle with line segment from $(1+(2 \alpha-1)|z|) /(1+|z|)$ to $(1-(2 \alpha-1)|z|) /(1-|z|)$ as a diameter. In this paper we consider a subclass of $\mathscr{S}_{\alpha}^{*}$ consisting of those $f(z)$ for which $\left|z f^{\prime}(z)\right| f(z)-1 \mid<1-\alpha$ for $|z|<1$ and denote it by $\mathscr{S}^{*}[\alpha]$. Sharp results concerning coefficients, distortion, and the radius of convexity are obtained. Furthermore, it is shown that $\sum_{n=2}^{\infty}[(n-\alpha) /(1-\alpha)]\left|a_{n}\right| \leqq 1$ is a sufficient condition for $f(z)$ to be in $\mathscr{S}^{*}[\alpha]$. Results about $\sum_{n=2}^{\infty} n\left|a_{n}\right| \leqq 1$ have previously been the subject of papers by Goodman [3], MacGregor [4], and Schild [6].

\section{Coefficient theorems.}

THEOREM 1. Let $f(z)=z+\sum_{n=2}^{\infty} a_{n} z^{n}$ and suppose that

$$
\sum_{n=2}^{\infty}[(n-\alpha) /(1-\alpha)]\left|a_{n}\right| \leqq 1 ;
$$

then $f(z) \in \mathscr{S}^{*}[\alpha]$ for $\alpha \in[0,1)$.

Proof. Let $|z|=1$, then

$$
\begin{aligned}
\left|z f^{\prime}(z)-f(z)\right|-(1-\alpha) & |f(z)| \\
& =\left|\sum_{n=2}^{\infty}(n-1) a_{n} z^{n}\right|-(1-\alpha)\left|z+\sum_{n=2}^{\infty} a_{n} z^{n}\right| \\
& \leqq \sum_{n=2}^{\infty}(n-\alpha)\left|a_{n}\right|-(1-\alpha) \leqq 0
\end{aligned}
$$

Received by the editors June 8, 1972.

AMS (MOS) subject classifications (1970). Primary 30A32.

Key words and phrases. Schlicht functions, starlike functions, radius of convexity.

(c) American Mathematical Society 1974 
by the hypothesis. Hence $\left|z f^{\prime}(z) / f(z)-1\right|<1-\alpha$ for $|z|<1$ by the maximum modulus theorem. We note that $f(z)=z-[(1-\alpha) /(n-\alpha)] z^{n}$ is an extremal function with respect to the theorem since $\left|z f^{\prime}(z)\right| f(z)-1 \mid=1-\alpha$ for $z=1, \alpha \in[0,1)$, and $n=2,3, \cdots$. We also observe that the converse to the theorem is false in that $f(z)=z e^{(1-\alpha) z} \in \mathscr{S}^{*}[\alpha]$ but

$$
\sum_{n=2}^{\infty} \frac{n-\alpha}{1-\alpha}\left|a_{n}\right|=\sum_{n=2}^{\infty} \frac{n-\alpha}{1-\alpha} \frac{(1-\alpha)^{n-1}}{(n-1) !}>2 e^{1-\alpha}-1>1 \text { for all } \alpha \in[0,1) \text {. }
$$

For $f(z)=z+\sum_{n=2}^{\infty} a_{n} z^{n} \in \mathscr{S}_{\alpha}^{*}$ it has been shown [7] that the sharp inequality $\left|a_{n}\right| \leqq \prod_{k=2}^{n}(k-2 \alpha) /(n-1)$ ! holds for $n=2,3, \cdots$ and is attained by $f(z)=z(1-z)^{-2(1-\alpha)}$. It is interesting to note that $(1-\alpha) /(n-1)$ appears as a factor of the upper bound for each $\left|a_{n}\right|$. For our class it is the upper bound.

THEOREM 2. Let $f(z)=z+\sum_{n=2}^{\infty} a_{n} z^{n} \in \mathscr{S}^{*}[\alpha]$, then $\left|a_{n}\right| \leqq(1-\alpha) /(n-1)$ for $n=2,3, \cdots$ and $\alpha \in[0,1)$.

Proof. If $f(z) \in \mathscr{S}^{*}[\alpha]$, then $\left|z f^{\prime}(z)\right| f(z)-1 \mid<1-\alpha$ and since the absolute value vanishes for $z=0$ we obtain

$$
z f^{\prime}(z) / f(z)=1+\omega(z)
$$

where $\omega(z)=\sum_{k=1}^{\infty} \omega_{k} z^{k}$ is analytic and $|\omega(z)|<1-\alpha$ for $|z|<1$. From (1) we see that $z f^{\prime}(z)-f(z)=f(z) \omega(z)$ or equivalently

$$
\sum_{k=2}^{\infty}(k-1) a_{k} z^{k}=\left(z+\sum_{k=2}^{\infty} a_{k} z^{k}\right)\left(\sum_{k=1}^{\infty} \omega_{k} z^{k}\right) .
$$

Equating coefficients on both sides of (2) shows that

$$
(n-1) a_{n}=\omega_{n-1}+\sum_{k=2}^{n-1} a_{1} \omega_{n-k} \text { for } n=2,3, \cdots
$$

which implies that the coefficient $a_{n}$ on the left side of (2) is dependent only on $a_{2}, a_{3}, \cdots, a_{n-1}$ on the right side of (2). Hence for $n \geqq 2$

$$
\sum_{k=2}^{n}(k-1) a_{k} z^{k}+\sum_{k=n+1}^{\infty} A_{k} z^{k}=\left(z+\sum_{k=2}^{n-1} a_{k} z^{k}\right) \omega(z)
$$

for a proper choice of $A_{k}$. Squaring the moduli of both sides of (3) and integrating around $|z|=r<1$ we get, using the fact that $|\omega(z)|<1-\alpha$ for $|z|<1$,

$$
\sum_{k=2}^{n}(k-1)^{2}\left|a_{k}\right|^{2} r^{2 k}+\sum_{k=n+1}^{\infty}\left|A_{k}\right|^{2} r^{2 k}<\left(1+\sum_{k=2}^{n-1}\left|a_{k}\right|^{2}\right)(1-\alpha)^{2} .
$$


Let $r \rightarrow 1$ and we find that

or

$$
\sum_{k=2}^{n}(k-1)^{2}\left|a_{k}\right|^{2} \leqq\left(1+\sum_{k=2}^{n-1}\left|a_{k}\right|^{2}\right)(1-\alpha)^{2}
$$

$$
(n-1)^{2}\left|a_{n}\right|^{2} \leqq(1-\alpha)^{2}-\sum_{k=2}^{n-1}\left[(k-1)^{2}-(1-\alpha)^{2}\right]\left|a_{k}\right|^{2} \leqq(1-\alpha)^{2}
$$

and it follows that $\left|a_{n}\right| \leqq(1-\alpha) /(n-1)$. This.proof is based on a technique found in Clunie and Keogh [2]. For sharpness, consider the function

$$
f(z)=z \exp [(1-\alpha) /(n-1)] z^{n-1}=z+[(1-\alpha) /(n-1)] z^{n}+\cdots .
$$

3. Distortion theorems. In order to obtain distortion properties of $f(z)$ and $f^{\prime}(z)$ we need the representation given by the following lemma.

LEMMA 1. $f(z) \in \mathscr{S}^{*}[\alpha]$ if and only if

$$
f(z)=z \cdot \exp \left\{\int_{0}^{z} \phi(t) d t\right\}
$$

where $\phi(z)$ is analytic for $|z|<1$ and $|\phi(z)| \leqq 1-\alpha$ for $|z|<1$ and $\alpha \in[0,1)$.

Proof. The "only if" is easily obtained by integrating (1) with $\omega(z)=$ $z \phi(z)$ while $f(z) \in \mathscr{S}^{*}[\alpha]$ follows from differentiation and simple manipulation.

Theorem 3. If $f(z) \in \mathscr{S}^{*}[\alpha]$, then

$$
|z| e^{(\alpha-1)|z|} \leqq|f(z)| \leqq|z| e^{(1-\alpha)|z|},
$$

and

(ii) $(1+(\alpha-1)|z|) e^{(\alpha-1)|z|} \leqq\left|f^{\prime}(z)\right| \leqq(1+(1-\alpha)|z|) e^{(1-\alpha)|z|}$.

Proof. Since

$$
\left|\int_{0}^{z} \phi(t) d t\right| \leqq \int_{0}^{|z|}|\phi(t)||d t| \leqq \int_{0}^{|z|}(1-\alpha)|d t|=(1-\alpha)|z|,
$$

then (i) follows by virtue of Lemma 1, and (ii) by applying the triangle inequality to $f^{\prime}(z)=(1+z \phi(z)) \cdot f(z) / z$. Both parts of the theorem are sharp for $f(z)=z e^{(1-\alpha) z}$.

4. The radius of convexity. It is well known that every univalent function maps $|z|<2-\sqrt{ } 3=0.267 \cdots$ onto a convex region and that for the class of starlike functions $\mathscr{S}_{0}^{*}$ this estimate cannot be improved since the extremal function for the class of univalent functions, the Koebe function $K(z)=z /\left(1-e^{i \theta} z\right)^{2}$, is also starlike. Here we determine the exact 
radius of convexity of $\mathscr{S}^{*}[\alpha]$ as a function of $\alpha$ for each $\alpha \in[0,1)$. In particular, it is shown that for $\mathscr{S}^{*}[0]$ the radius of convexity is $(3-\sqrt{ } 5) / 2=0.382 \cdots$.

THEOREM 4. Suppose that $f(z) \in \mathscr{S}^{*}[\alpha]$; then $f(z)$ maps $|z|<r$ onto a convex domain for

$$
r=(3-\sqrt{ } 5) /(2-2 \alpha), \quad \text { if } \alpha \in\left[0, \alpha_{0}\right],
$$

and

$$
\begin{array}{r}
r=\left[\left(\left(-2 \alpha^{2}+\alpha-1\right)+2 \alpha\left(6-6 \alpha+\alpha^{2}\right)^{1 / 2}\right) /\left(1+3 \alpha-4 \alpha^{2}\right)\right]^{1 / 2}, \\
\text { if } \alpha \in\left[\alpha_{0}, 1\right),
\end{array}
$$

where $\alpha_{0}=1-(1+\sqrt{ } 6)(3 \sqrt{ } 5-5) / 10=0.411 \cdots$. The result is sharp.

Proof. As a notational convenience let $\beta=1-\alpha$. In [1] it is shown that if $\phi(z)$ is analytic for $|z|<1$ with $|\phi(z)| \leqq 1$, then

$$
\left|\phi^{\prime}(z)\right| \leqq\left(1-|\phi(z)|^{2}\right) /\left(1-|z|^{2}\right) .
$$

If, however, $|\phi(z)| \leqq \beta$ then we may apply the previous result to $\phi(z) / \beta$ and obtain

$$
\left|\phi^{\prime}(z)\right| \leqq\left(\beta-|\phi(z)|^{2} \beta^{-1}\right) /\left(1-|z|^{2}\right) .
$$

Let $\omega(z)=-z \phi(z)$ in (1); then after taking the logarithmic derivative of both sides we have

$$
\operatorname{Re}\left\{\frac{z f^{\prime \prime}(z)}{f^{\prime}(z)+1}\right\}=\operatorname{Re}\left\{(1-z \phi(z))-\frac{z^{2} \phi^{\prime}(z)+z \phi(z)}{1-z \phi(z)}\right\} .
$$

Regrouping and then applying (4) and the triangle inequality to the right side of (5) gives us

$$
\begin{aligned}
& \operatorname{Re}\left\{z f^{\prime \prime}(z) / f^{\prime}(z)+1\right\} \geqq 1-(|z \phi(z)|\left(1+(1-|z \phi(z)|)^{-1}\right) \\
&\left.+|z|^{2}\left|\phi^{\prime}(z)\right|(1-|z \phi(z)|)^{-1}\right) \\
& \geqq 1-(|z \phi(z)|(2-|z \phi(z)|)\left(1-|z|^{2}\right) \\
&\left.+|z|^{2}\left(\beta-|\phi(z)|^{2} \beta^{-1}\right)\right) \\
& \cdot\left((1-|z \phi(z)|)\left(1-|z|^{2}\right)\right)^{-1} .
\end{aligned}
$$

For $f(z)$ to be convex in $|z|<r$ it suffices to have $\operatorname{Re}\left\{z f^{\prime \prime}(z) \mid f^{\prime}(z)+1\right\}>0$ in $|z|<r$, see [5]. In our case, (5) will certainly be positive when the right side of (6) is positive which is whenever

$$
\begin{aligned}
\beta|z \phi(z)|(2-|z \phi(z)|)\left(1-|z|^{2}\right)+|z|^{2}\left(\beta^{2}-|\phi(z)|^{2}\right) & \\
& <\beta(1-|z \phi(z)|)\left(1-|z|^{2}\right) .
\end{aligned}
$$


In order to discuss (7) let us consider the function

$$
p(x)=\left[\left(1-r^{2}\right) \beta+1\right] x^{2}-3\left(1-r^{2}\right) \beta x-\left(\beta^{2} r^{2}-\beta\left(1-r^{2}\right)\right)
$$

where $r=|z| \in[0,1)$ and $x=|z \phi(z)| \in[0, r \beta]$. Clearly

$$
p^{\prime}(x)=2\left[\left(1-r^{2}\right) \beta+1\right] x-3\left(1-r^{2}\right) \beta=0
$$

for $x=x_{0}=3\left(1-r^{2}\right) \beta /\left(2\left(\left(1-r^{2}\right) \beta+1\right)\right)$ and $p^{\prime \prime}(x)>0$. Hence, for each $\beta, p(x)$ is a parabola opening upward. Thus an investigation of (7) now reduces to trying to determine some relationship among $\beta, r$, and $x$ so that $p(x)>0$. In order to do so we explore the geometric significance of the two cases: (a) $p^{\prime}(r \beta) \leqq 0$ and (b) $p^{\prime}(r \beta) \geqq 0$.

(a) If $p^{\prime}(r \beta) \leqq 0$, then $r \beta \leqq x_{0}$ and so $p(x) \geqq p(r \beta)$ for $x \in[0, r \beta]$. Now, let $p(r \beta)$ be considered as a function of $r$ with $\beta$ held constant, then (7) will be satisfied when

$$
p(r \beta)=\beta\left(1-r^{2}\right)\left(1-3 \beta r+\beta^{2} r^{2}\right)>0
$$

which is exactly when $r<(3-\sqrt{ } 5) /(2 \beta)$. This result, however, is valid only for those $\beta$ for which $r \beta \leqq x_{0}$ or equivalently, for $\beta$ satisfying

$$
2 \sqrt{ } 5 \beta^{2}+(2 \sqrt{ } 5-6) \beta+(15-7 \sqrt{ } 5) \geqq 0 .
$$

Hence for $\beta \in\left[\beta_{0}, 1\right]$ where $\beta_{0}=(1+\sqrt{ } 6)(3 \sqrt{ } 5-5) / 10$. The result is proved sharp by choosing the function $f(z)=z e^{\beta z}$ for $\beta \in\left[\beta_{0}, 1\right]$.

(b) On the other hand, $p^{\prime}(r \beta) \geqq 0$ implies $r \beta \geqq x_{0}$ and $p(x) \geqq p\left(x_{0}\right)$ for $x \in[0, r \beta]$. Again let $\beta$ be fixed. Now (7) will be satisfied when

$$
\begin{aligned}
p\left(x_{0}\right)=-\beta\left(\left(5 \beta-4 \beta^{2}\right) r^{4}+2\left(2 \beta^{2}-3 \beta+2\right) r^{2}\right. & +(5 \beta-4)) \\
\cdot & \left(4\left(\left(1-r^{2}\right) \beta+1\right)\right)^{-2}>0
\end{aligned}
$$

which is whenever

$r<r_{0}=\left[\left(-\left(2 \beta^{2}-3 \beta+2\right)+2(1-\beta)\left(\beta^{2}+4 \beta+1\right)^{1 / 2}\right) /\left(5 \beta-4 \beta^{2}\right)\right]^{1 / 2}$ provided that $r \beta \geqq x_{0}$, that is, for $\beta \in\left(0, \beta_{0}\right]$.

We conclude with an existence proof for a sharp function. Suppose that $\psi(z)=\beta\left(z-z_{0}\right) /\left(1-z_{0} z\right)$ where $z_{0}$ is real; then

and so

$$
z_{0}=(\psi(z)-\beta z) /(z \psi(z)-\beta)
$$

$$
\psi^{\prime}(z)=\beta\left(1-z_{0}^{2}\right) /\left(1-z_{0} z\right)^{2}=\left(\beta^{2}-\psi^{2}(z)\right) /\left(\beta\left(1-z^{2}\right)\right) .
$$

If we now let $z f^{\prime}(z) / f(z)=1-z \psi(z)$, then

$$
\begin{aligned}
& z f^{\prime \prime}(z) / f^{\prime}(z)+1 \\
& \quad=\left(\left(\left(1-z^{2}\right) \beta+1\right)(z \psi(z))^{2}-3\left(1-z^{2}\right) \beta(z \psi(z))\right. \\
& \left.\quad-\left(\beta^{2} z^{2}-\beta\left(1-z^{2}\right)\right)\right) /\left(\beta(1-z \psi(z))\left(1-z^{2}\right)\right)=0
\end{aligned}
$$


when $z=r_{0}$ and $z \psi(z)=r_{0} \psi\left(r_{0}\right)=x_{0}$. Since $x_{0} \leqq r_{0} \beta$ we have

$$
r_{0} \psi\left(r_{0}\right)=r_{0} \beta\left(r_{0}-z_{0}\right) /\left(1-r_{0} z_{0}\right) \leqq r_{0} \beta
$$

and so $\left(r_{0}-z_{0}\right) /\left(1-r_{0} z_{0}\right) \leqq 1$ which implies that $\left|z_{0}\right| \leqq 1$. Hence $|\psi(z)| \leqq \beta$ for $|z|<1$ and so by Lemma 1

$$
f(z)=z \cdot \exp \left\{\int_{0}^{z}\left(-\beta\left(t-z_{0}\right) /\left(1-z_{0} t\right)\right) d t\right\} \in \mathscr{S}^{*}[1-\beta] .
$$

\section{REFERENCES}

1. C. Carathéodory, Theory of functions of a complex variable. Vol. 2, Chelsea, New York, 1954. MR 16, 346.

2. J. Clunie and F. R. Keogh, On starlike and convex schlicht functions, J. London Math. Soc. 35 (1960), 229-233. MR 22 \#1682.

3. A. W. Goodman, Univalent functions and nonanalytic curves, Proc. Amer. Math. Soc. 8 (1957), 598-601. MR 19, 260.

4. T. H. MacGregor, A class of univalent functions, Proc. Amer. Math. Soc. 15 (1964), 311-317. MR 28 \#2206.

5. Z. Nehari, Conformal mapping, McGraw-Hill, New York, 1952. MR 13, 640.

6. A. Schild, On a class of functions schlicht in the unit circle, Proc. Amer. Math. Soc. 5 (1954), 115-120. MR 15, 694.

7. - On starlike functions of order $\alpha$, Amer. J. Math. 87 (1965), 65-70. MR 30 \#4929.

Department of Mathematics, la Salle College, Philadelphia, Pennsylvania 19141 